\title{
Stent experiences in emergency conditions in acute mechanical intestinal obstruction caused by colorectal cancer
}

\author{
Savas Bayrak ${ }^{1}$, Cihad Tatar ${ }^{2}$, Erdem Kinaci², Ekrem Cakar², Sukru Colak², Mert Mahsuni Sevinc ${ }^{2}$, Hasan Bektas ${ }^{2}$ \\ ${ }^{1}$ Department of General Surgery, Bakırköy Dr. Sadi Konuk Training and Research Hospital, Istanbul, Turkey \\ 2Department of General Surgery, Istanbul Training and Research Hospital, Istanbul, Turkey
}

Videosurgery Miniinv 2019; 14 (1): 46-51

DOI: https://doi.org/10.5114/wiitm.2018.76072

\begin{abstract}
Introduction: Stent treatment can be applied to avoid surgery in surgically risky patients or to turn a high-risk emergency operation into a lower-risk elective operation and save time.

Aim: In this study, the techniques, clinical efficacy, safety and complications of endoscopic stents applied in emergency conditions were evaluated in patients with acute mechanical intestinal obstruction (AMIO) due to colorectal cancer.

Material and methods: Between 2013 and 2015, 23 patients with an average age of $69.5 \pm 13.5$ years who presented with AMIO and anastomosis stenosis secondarily to cancer to the emergency department were subjected to stent treatment under emergency conditions.

Results: Thirteen (56.5\%) patients were diagnosed with colon cancer, 5 (21.7\%) with rectal cancer, and 5 (21.7\%) with stenosis in the previous anastomosis line. Fourteen (60.9\%) patients were diagnosed with stage 4 cancer, 7 (30.4\%) with stage 3 cancer and 2 (8.7\%) with stage 2 cancer. The stents were applied to the sigmoid colon in $10(43.5 \%)$ patients, to the recto-sigmoid area in $9(39.1 \%)$ patients and to the rectum area in $4(17.4 \%)$ patients. While 14 (60.9\%) patients had local or locally advanced disease, 9 (39.1\%) patients had metastases in different parts of their bodies, particularly in their livers.

Conclusions: The study demonstrates that stents offer a favorable therapeutic alternative to emergency surgery and are associated with promising short-term outcomes as well as an acceptable safety profile for AMIO.
\end{abstract}

Key words: colonoscopy, stenting, colonic obstruction.

\section{Introduction}

Colorectal cancer is one of the five most common cancers in the world. Surgery is the primary treatment of this type of cancer. The important points in the surgery are proper dissection, resection and adequate lymph node dissection. Despite the high morbidity and mortality rates, recent innovations have significantly increased survival in colorectal cancer $[1,2]$. Acute intestinal obstruction occurs in $8-29 \%$ of patients with colorectal cancer. These patients have locally advanced disease or metastases that often make resection impossible [3]. Hartmann's procedure is the best approach for the surgical resolution of the obstruction due to having a lower mortality rate compared to other options [4]. Endoscopic stent treatment is an alternative treatment method used in surgically risky patients or to turn a high-risk emergency operation into a lower-risk elective operation [5, 6]. Since the introduction of colonic stenting, the endoscopic approach has been advocated as an alternative to conventional surgery for relieving acute colorectal obstruction, whether palliative or as a bridge to definitive surgery. With endoscopic stent treatment, the 
obstruction is removed from the middle to provide rehabilitation of the intestine. During this time planning is ensured for imaging, surgery, and chemotherapy and radiotherapy treatment for staging. In addition, a single-step resection and reanastomosis is also available in appropriate patients [6].

\section{Aim}

In this study, the techniques, clinical efficacy, safety and complications of the endoscopic stents applied in emergency conditions were evaluated in patients with acute mechanical intestinal obstruction (AMIO) due to colorectal cancer.

\section{Material and methods}

The compliance of the study protocol with the principles of the Helsinki Declaration was approved by the Research Ethics Committee of Istanbul Education and Research Hospital. Between 2010 and 2015, 23 patients with an average age of $69.5 \pm 13.5$ years who presented with $\mathrm{AMIO}$ and anastomosis stenosis secondarily to cancer to the emergency department were subjected to stent treatment under emergency conditions. Patients who had not had bowel movements during the last $24 \mathrm{~h}$, those with abdominal distension, fecaloid vomiting, and those who have enlarged colonic loops on abdominal radiography were accepted as having AMIO (physical examination, blood tests, abdominal X-ray and computed tomography (CT) scan or magnetic resonance imaging $(\mathrm{MRI}))$. The self-expanding cross stents were placed in all patients endoscopically under endoscopic and fluoroscopic observation within $24 \mathrm{~h}$ following their admission. Before placement of the stent, the area under the obstruction of the colon or rectum was cleaned with a rectal enema. No patient was given an oral laxative. All stenting procedures were performed in the left lateral decubitus position. All patients were premedicated with 2-4 mg of intravenous (i.v.) midazolam and 25-50 mg of pethidine hydrochloride before the procedure. A water soluble contrast agent was injected to calculate the length, proximal extension and diameter of the stenosis. Under fluoroscopic guidance, 60-120 mm long and 20-30 mm diameter coated and uncoated stents were placed in the stenosis zone through a colonoscope. Technical success was defined as successful placement of the stent. Clinical success was considered as technically successful stent placement followed by the development of colonic decompression within $24 \mathrm{~h}$ without any additional endoscopic or surgical intervention. Although antibiotic prophylaxis is not routinely performed, all patients with antibiotic prophylaxis due to AMIO have been treated with cefuroxime axetil plus metronidazole or ampicillin sulbactam because the inflation of the colon with air during the procedure carries the risk of perforation or development of bacteremia. Descriptive statistical methods were used for the study.

\section{Results}

Twelve (52\%) male and 11 (48\%) female, in total 23 patients with a mean age of $69.5 \pm 13.5$ years and with AMIO diagnoses were implemented with an endoscopic stent under emergency conditions (Tables I and II). Thirteen (56.5\%) patients were diagnosed with colon cancer (C-Ca), 5 (21.7\%) with rectum cancer (R-Ca) and 5 (21.7\%) with anastomotic stricture (AS). Systematic diseases (diabetes, hypertension) were observed in 16 (69.6\%) patients. The American Society of Anesthesiologists (ASA) score was determined to be 3 in 14 patients (60.9\%), 2 in 6 patients (26.1\%) and 1 in 3 patients (13.0\%). Fourteen (60.9\%) patients were diagnosed with stage 4 cancer, 7 (30.4\%) with stage 3 cancer and 2 (8.7\%) with stage 2 cancer. While 11 (47.8\%) patients were followed up in our clinic following the stent application, 9 (39.1\%) patients were followed up by the oncology clinic, and $3(13.0 \%)$ patients left the follow-up process of their own volition. Stents were applied to the sigmoid colon (SC) in 10 (43.5\%) patients, to the recto-sigmoid (RS) area in 9 (39.1\%) patients, and to the rectum in $4(17.4 \%)$ patients. The lengths of the stents applied were $100 \mathrm{~mm}$ in 12 (52.2\%) patients, $80 \mathrm{~mm}$ in $6(26.1 \%)$ patients, $60 \mathrm{~mm}$ in $3(13.0 \%)$ patients and $120 \mathrm{~mm}$ in $2(8.7 \%)$ patients. While 14 (60.9\%) patients had local or locally advanced disease, 9 (39.1\%) patients had metastases in different parts of their bodies, particularly in their livers. Complications developed only in 3 (13.0\%) patients. Perforation was observed in 1 patient during the operation, and in 1 patient on the eighth day after the second stent application. The stent applied in 1 patient fell to the rectum on the eighth day. While $5(21.7 \%)$ patients underwent pre-stent dilatation, only 1 (4.3\%) patient underwent post-stent dilatation. Coated stents were applied to 9 (39.1\%) patients, while uncoated stents were applied to $14(60.9 \%)$ patients. Two or more stents were ap- 


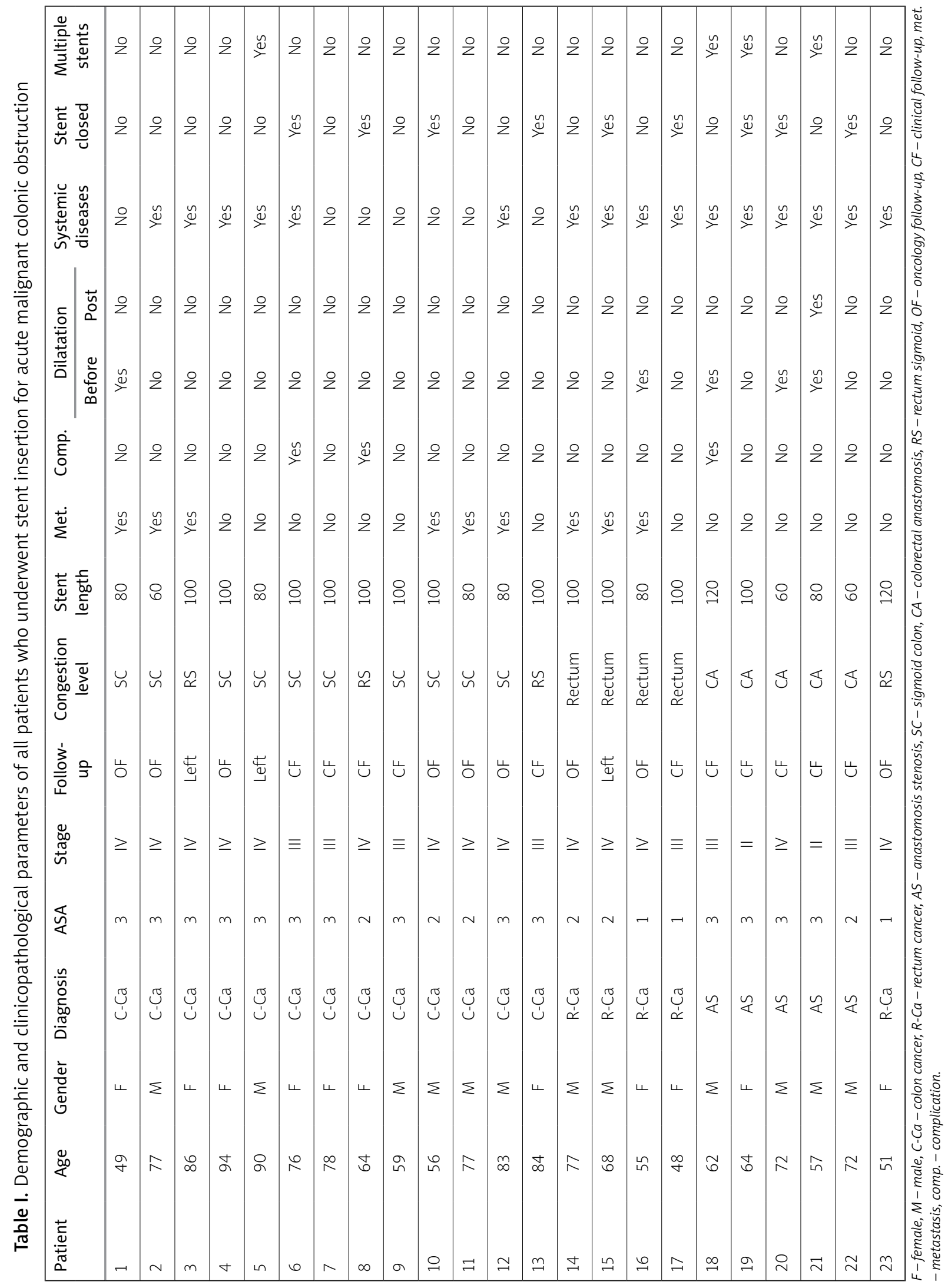


Table II. Details of demographic and clinicopathological parameters

\begin{tabular}{|c|c|}
\hline Parameter & Value \\
\hline \multicolumn{2}{|l|}{ Age [years]: } \\
\hline Mean \pm SD & $69.5 \pm 13.5$ \\
\hline Median (min.-max.) & $72(48-94)$ \\
\hline \multicolumn{2}{|l|}{ Gender: } \\
\hline Female & $11(47.8)$ \\
\hline Male & $12(52.2)$ \\
\hline \multicolumn{2}{|l|}{ Diagnosis: } \\
\hline AS & $5(21.7)$ \\
\hline $\mathrm{C}-\mathrm{Ca}$ & $13(56.5)$ \\
\hline $\mathrm{R}-\mathrm{Ca}$ & $5(21.7)$ \\
\hline \multicolumn{2}{|l|}{ ASA: } \\
\hline 1 & $3(13.0)$ \\
\hline 2 & $6(26.1)$ \\
\hline 3 & $14(60.9)$ \\
\hline \multicolumn{2}{|l|}{ Stage: } \\
\hline$\|$ & $2(8.7)$ \\
\hline III & $7(30.4)$ \\
\hline IV & $14(60.9)$ \\
\hline \multicolumn{2}{|l|}{ Follow-up: } \\
\hline CF & $11(47.8)$ \\
\hline Left & $3(13.0)$ \\
\hline OF & $9(39.1)$ \\
\hline \multicolumn{2}{|l|}{ Congestion level: } \\
\hline CA & $5(21.7)$ \\
\hline Rectum & $4(17.4)$ \\
\hline RS & $4(17.4)$ \\
\hline SC & $10(43.5)$ \\
\hline
\end{tabular}

\begin{tabular}{|c|c|}
\hline Parameter & Value \\
\hline \multicolumn{2}{|c|}{ Stent length: } \\
\hline 100 & $12(52.2)$ \\
\hline 120 & $2(8.7)$ \\
\hline 60 & $3(13.0)$ \\
\hline 80 & $86(26.1)$ \\
\hline \multicolumn{2}{|l|}{ Met.: } \\
\hline No & $14(60.9)$ \\
\hline Yes & $9(39.1)$ \\
\hline \multicolumn{2}{|l|}{ Comp.: } \\
\hline No & $20(87.0)$ \\
\hline Yes & $3(13.0)$ \\
\hline \multicolumn{2}{|l|}{ Dilatation: } \\
\hline \multicolumn{2}{|l|}{ Before: } \\
\hline No & $18(78.3)$ \\
\hline Yes & $5(21.7)$ \\
\hline \multicolumn{2}{|l|}{ Post: } \\
\hline No & $22(95.7)$ \\
\hline Yes & $1(4.3)$ \\
\hline \multicolumn{2}{|c|}{ Systemic diseases: } \\
\hline No & $7(30.4)$ \\
\hline Yes & 16 (69.6) \\
\hline \multicolumn{2}{|c|}{ Stent closed: } \\
\hline No & $14(60.9)$ \\
\hline Yes & $9(39.1)$ \\
\hline \multicolumn{2}{|c|}{ Multiple stents: } \\
\hline No & 19 (82.6) \\
\hline Yes & $4(17.4)$ \\
\hline
\end{tabular}

$C$-Ca - colon cancer, $R$-Ca - rectum cancer, AS - anastomosis stenosis, SC - sigmoid colon, CA - colorectal anastomosis, RS - rectum sigmoid, OF - oncology follow-up, CF-clinical follow-up, met. - metastasis, comp. - complication.

plied in 4 (17.4\%) patients (Table I). After ensuring sufficient decompression of the colon (in a case requiring chemoradiotherapy followed by neoadjuvant therapy), elective resection was performed in 1 patient with indications. The mean follow-up period of the patients was 43 months (min.: 36, max.: 64), and the 3 -year survival rate and the overall survival rate were $34.8 \%$ and $21.7 \%$, respectively.

\section{Discussion}

Stenting of AMIO is an effective option for patients presenting with obstructive colorectal cancer, in which decompression is required to perform an elective one-stage oncologic surgical resection. This approach may also offer palliation for patients who are not candidates for operative intervention, and it is associated with a lower mortality and shorter hospital stay $[7,8]$.

Acute mechanical intestinal obstruction is observed in approximately a quarter of colorectal cancer cases. The two most common approaches to these patients are colostomy and resection of the primary tumor if appropriate. Most patients are comorbid, malnourished, dehydrated, and elderly. The morbidity and mortality of surgery under emergency conditions are about $30-40 \%$ and $7-15 \%$, respec- 
tively [9-11]. ASA values were detected as 2 and 3 in $20(87 \%)$ of the patients in this study. Of these patients, 16 (69.6\%) had systemic diseases (diabetes, hypertension, etc.). In addition, 9 of the patients (39.1\%) had metastases in other organs. Colonic stents are associated with lower mortality and morbidity compared to emergency surgery in cases of AMIO. In patients with multiple medical comorbidities, poor performance status and increased ASA, a colonic stent can be considered as an alternative option as a bridge to surgery.

Patients may be appropriately staged following the stent application and chemoradiotherapy or elective surgery may be recommended. In addition, stents can be easily used in the palliation of inoperable obstructive colorectal malignancies or extrinsic compression. Technical and clinical success rates were reported between $94-96.5 \%$ and $91-100 \%$, respectively, when the feasibility, safety and efficacy of stents were evaluated in various retrospective studies [12-17] In this study, stents were applied to the SC area in $10(43.5 \%)$ patients, to the RS area in $9(39.1 \%)$ patients, and to the rectum area in $4(17.4 \%)$ patients. The length of the stents was $100 \mathrm{~mm}$ in $12(52.2 \%)$ patients, $80 \mathrm{~mm}$ in $6(26.1 \%)$ patients, $60 \mathrm{~mm}$ in 3 $(13.0 \%)$ patients and $120 \mathrm{~mm}$ in $2(8.7 \%)$ patients. No complication was observed following the stent application in $20(87.0 \%)$ patients and the procedure was successful. Perforations developed only in $2(8.69 \%)$ patients. The stents are placed with the support of an endoscope. If the area of obstruction cannot be passed by the endoscope, balloon dilatation is applied. Colorectal perforation is one of the most feared complications of stent placement. The perforation most commonly occurs in the rectum-sigmoid region, particularly in the rectosigmoid junction. Patients with balloon pre-dilatation have a higher probability of perforation. Some authors do not recommend pre-stent balloon dilatation. The stent placement was performed with the support of an endoscope in cases in this study. Balloon dilatation was applied in 6 patients where a stent could not be passed due to stenosis. Of these patients, 5 (21.7\%) were recanalized with balloon dilatation before stenting and $1(4.3 \%)$ after stenting, and then the stents were placed successfully. Perforation occurred in $1(16.6 \%)$ patient who underwent balloon dilatation.

Today, effective chemotherapeutic agents have been introduced in colorectal cancers. The stent placement shortens the time to start chemothera- py. Patients who cannot receive chemotherapy because of surgery and complications are able to have this opportunity with stent application. Stenting is the most appropriate treatment to use in palliative treatment because the mean survival for advanced colorectal cancer is between 119 and 150 days [18, 19]. Of the patients in this study, $18(78.3 \%)$ had colon cancer, and 5 (21.7\%) had rectal cancer. Fourteen $(60.9 \%)$ were diagnosed with stage 4 cancer, 7 (30.4\%) with stage 3 cancer and 2 (8.7\%) with stage 2 cancer. The mean follow-up period of the patients was 43 months (min.: 36, max.: 64), and the 3-year survival rate and the overall survival rate were $34.8 \%$ and $21.7 \%$, respectively. In the study of Gibor et al. [20], which applied self-expanding metal stents in 21 patients, the 4-year survival rate was $52.4 \%$ and overall survival was $33.6 \%$. The proportion of patients in stage 4 out all patients was $24 \%$ according to Gibor et al., but $60.9 \%$ in this study. We think our overall survival rates are lower because of the majority of stage 4 patients in our study.

\section{Conclusions}

Acute mechanical intestinal obstruction poses significant challenges in management due to the frailty of the presenting patients and the high mortality/morbidity rates associated with emergency surgery. Although our study suffers from a small sample size, it demonstrates that stents offer a favorable therapeutic alternative to emergency surgery and are associated with promising short-term outcomes as well as an acceptable safety profile. Larger scale studies looking at long-term survival and oncological outcomes are awaited.

\section{Conflict of interest}

The authors declare no conflict of interest.

\section{References}

1. Ferlay J, Shin HR, Bray F, et al. Globocan 2008, Cancer Incidence And Mortality Worldwide: IARC Cancer Base No. 10. Lyon, France: IARC; 2010.

2. Brenner H, Kloor M, Pox CP. Colorectal cancer. Lancet 2014; 383: 1490-502.

3. Dulucq JL, Wintringer P, Beyssac R, et al. One-stage laparoscopic colorectal resection after placement of self-expanding metallic stents for colorectal obstruction: a prospective study. Dig Dis Sci 2006; 51: 2365-71.

4. Useros JV, Foncillas JG. Obesity and colorectal cancer: molecular features of adipose tissue. J Transl Med 2016; 14: 21. 
5. Moug SJ, McCarthy K, Coode-Bate J, et al. Laparoscopic versus open surgery for colorectal cancer in the older person: a systematic review. Ann Med Surg 2015; 4: 311-8.

6. Sagar J. Role of colonic stents in the management of colorectal cancers. World J Gastrointest Endosc 2016; 25: 198-204.

7. Small AJ, Young-Fadok TM, Baron TH. Expandable metal stent placement for benign colorectal obstruction: outcomes for 23 cases. Surg Endosc 2008; 22: 454-62.

8. Takahashi H, Okabayashi K, Tsuruta M, et al. Self-expanding metallic stents versus surgical intervention as palliative therapy for obstructive colorectal cancer: a meta-analysis. World J Surg 2015; 39: 2037-44.

9. Huang X, Lv B, Zhang S, Meng L. Preoperative colonic stents versus emergency surgery for acute left-sided malignant colonic obstruction: a meta-analysis. J Gastrointest Surg 2014; 18: 584-91.

10. Hong SP, Kim TI. Colorectal stenting: an advanced approach to malignant colorectal obstruction. World J Gastroenterol 2014; 20: 16020-8

11. Small AJ, Coelho-Prabhu N, Baron TH. Endoscopic placement of self expandable metal stents for malignant colonic obstruction: long-term outcomes and complication factors. Gastrointest Endosc 2010; 71: 560-72.

12. Khot UP, Lang AW, Murali K, et al. Systematic review of the efficacy and safety of colorectal stents. Br J Surg 2002; 89: 1096-102.

13. Sebastian S, Johnston S, Geoghegan T, et al. Pooled analysis of the efficacy and safety of self-expanding metal stenting in malignant colorectal obstruction. Am J Gastroenterol 2004; 99: 2051-7.

14. Dionigi G, Villa F, Rovera F, et al. Colonic stenting for malignant disease: review of literature. Surg Oncol 2007; 16: 153-5.

15. Han SH, Lee JH. Colonic stent related complications and their management. Clin Endosc 2014; 47: 415-9.

16. Stankiewicz R, Kozieł S, Pertkiewicz J, Zieniewicz K. Outcomes and complications of self-expanding metal stent placement for malignant colonic obstruction in a single-center study. Videosurgery Miniinv 2018; 13: 53-6.

17. Keswani RN, Azar RR, Edmundowicz SA, et al. Stenting for malignant colonic obstruction: a comparison of efficacy and complications in colonic versus extracolonic malignancy. Gastrointest Endosc 2009; 69: 675-80.

18. Debnath D. Comparison of stenting with emergency surgery as palliative treatment for obstructing primary left-sided colorectal cancer. Br J Surg 2004; 91: 511.

19. Borowiec AM, Wang CS, Yong E, et al. Colonic stents for colorectal cancer are seldom used mainly for palliation of obstruction: a population-based study. Can J Gastroenterol Hepatol 2016; 2016: 1945172.

20. Gibor U, Perry Z, Tirosh D, et al. Comparison of the long-term oncological outcomes of stent as a bridge to surgery and surgery alone in malignant colonic obstruction. Isr Med Assoc J 2017; 19: 736-40.

Received: 20.02.2018, accepted: 10.05.2018 